\title{
Dysregulation of upstream binding factor-1 acetylation at K352 is linked to impaired ribosomal DNA transcription in Huntington's disease
}

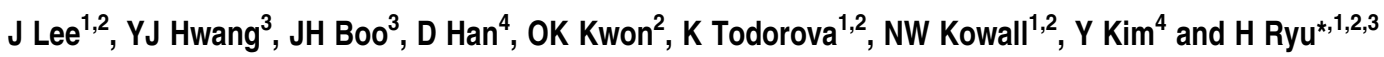

Huntington's disease (HD) is an autosomal-dominant neurological disorder caused by expanded CAG repeats in the Huntingtin (Htt) gene, but it is not known how this mutation causes neurodegeneration. Herein, we found that dysfunction of upstream binding factor-1 (UBF-1) is linked to reduced ribosomal DNA ( $\mathrm{rDNA}$ ) transcription in HD. We identified that UBF1 acetylation at Lys (K) 352 by CREB binding protein (CBP) is crucial for the transcriptional activity of rDNA. UBF1 mutation (K352A, K352Q, and K352R) decreased rDNA transcriptional activity. Moreover, both CBP-dHAT mutant and knockdown of CBP by siRNA reduced acetylation of UBF1 and resulted in the decreased transcription of rDNA into rRNA. ChIP analysis showed a significant reduction of UBF1 occupancy in the promoter of rDNA in STHdh${ }^{Q 111}$ cell line model of HD. These results demonstrate that abnormal activity of UBF1 and its acetylation by CBP are linked to impaired rDNA transcription in HD. This novel mechanism suggests that modulation of UBF-mediated rDNA synthesis by CBP may be a therapeutic target for improving neuronal rDNA transcription in HD.

Cell Death and Differentiation (2011) 18, 1726-1735; doi:10.1038/cdd.2011.38; published online 6 May 2011

Huntington's disease (HD) is an incurable and fatal autosomal dominant neurodegenerative disorder, typically of mid-life onset, characterized by a triad of chorea, cognitive abnormalities, and progressive dementia. The motor symptoms of HD are largely a consequence of profound neurodegeneration affecting the striatum. ${ }^{1} \mathrm{HD}$ is caused by an expansion of CAG repeats coding for glutamine $(Q)$ in exon 1 of the Huntingtin (Htt) gene. One mechanism by which mutant $\mathrm{Htt}$ (mtHtt) contributes to neurodegeneration is through transcriptional dysregulation and chromatin remodeling. ${ }^{2}$ Indeed, polyQ stretches in $\mathrm{mtH}$ tt interact physically with CBP (CREB binding protein), a transcriptional coactivator, and block the intrinsic histone acetyltransferase (HAT) activity of CBP. ${ }^{3}$ These specific interactions show that $\mathrm{mtH}$ tt modulates transcriptional signaling cascades that may initiate a number of downstream pathophysiological mechanisms relevant to HD.

The nucleolus is an important subnuclear component of the transcription machinery of ribosomal genes. ${ }^{4}$ The ribosomal DNA ( $r D N A$ ) encoding ribosomal RNA ( $r R N A$ ) is organized as tandem repeats, and is transcribed into $47 \mathrm{~S}$ precursor $\mathrm{rRNA}$ by a specialized transcription complex, consisting of RNA polymerase I and other co-regulatory factors. ${ }^{5}$ Neurons are postmitotic cells that have prominent nucleoli, but the role of this structure and the regulatory mechanism of rDNA remain unknown. ${ }^{6}$ RNA polymerase I and upstream binding factor (UBF) have critical roles in the formation of active nucleolar organizer regions, and maintenance of rRNA transcriptional activity. ${ }^{7}$ UBF is a member of the high mobility group (HMG) protein family and contains six HMG box DNA binding motifs. It is a highly conserved protein and there is UBF1 and UBF2 polypeptides, 97 and $94 \mathrm{kDa}$, respectively. ${ }^{8}$ Not only UBF is a RNA polymerase I factor essential for rDNA (genes encoding rRNAs in eukaryotes) transcription by inducing remodeling of ribosomal gene chromatin, but it also serves a structural role by binding to other sequences across the entire rDNA repeat. ${ }^{4}$ Interestingly, nucleolar accessory bodies (Cajal bodies) are associated with disorders caused by expansions of CAG repeats within genes, including $\mathrm{HD}$, and the neuronal nuclear inclusions characteristic of polyQ diseases show a marked association with Cajal bodies. ${ }^{9}$ In our study, we show for the first time that transcriptional modulation of rDNA, a key process in ribosome biogenesis, is altered in HD and that UBF and $C B P$ have a role in altered rRNA expression. We found that UBF1 protein levels and UBF-mediated transcriptional activity of rDNA are impaired in cellular and animal model of HD. In parallel, we determined that CBP interacts with UBF1, acetylates UBF1 at Lys $(\mathrm{K}) 352$, and modulates rDNA transcription.

\section{Results}

UBF1 is dysregulated in an animal model of HD. We examined UBF1 immunoreactivity, protein, and mRNA levels in the R6/2 transgenic mouse model of HD (Figure 1). The $\mathrm{R} 6 / 2$ line is a transgenic mouse line expressing exon 1 of the

\footnotetext{
${ }^{1}$ VA Boston Healthcare System, Boston, MA, USA; ${ }^{2}$ Department of Neurology, Boston University School of Medicine, Boston, MA, USA; ${ }^{3}$ World Class University Neurocytomics Group, Department of Biomedical Sciences, Seoul National University College of Medicine, Seoul, Republic of Korea and ${ }^{4}$ Department of Biomedical Sciences, Seoul National University College of Medicine, Seoul, Republic of Korea

*Corresponding author: H Ryu, Department of Neurology, Boston University School of Medicine, Building 1A, Rm109, Boston VA Healthcare System, 150 S. Huntington Avenue, Boston, MA 02130, USA. Tel: 857-364-5910; Fax: 857-364-4540; E-mail: hoonryu@bu.edu

Keywords: UBF1; rDNA transcription; CBP; acetylation; Huntington's disease

Abbreviations: ENH, enhancer; UCE, upstream control element; CORE, core region; ETS, external transcribed spacer; 5-FU, 5-fluorouracil

Received 06.10.10; revised 23.2.11; accepted 15.3.11; Edited by N Bazan; published online 06.5.11
} 

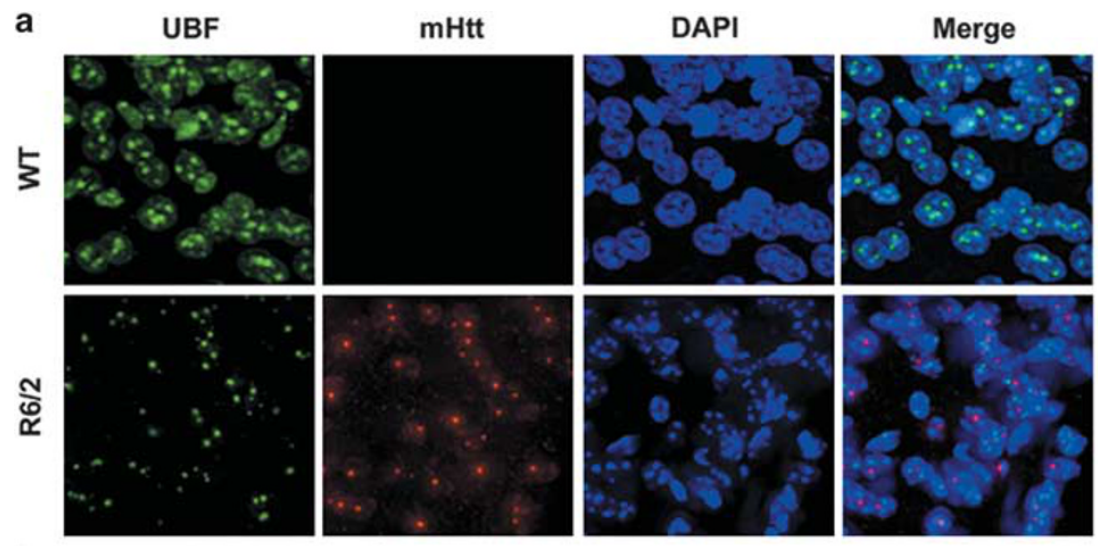

b
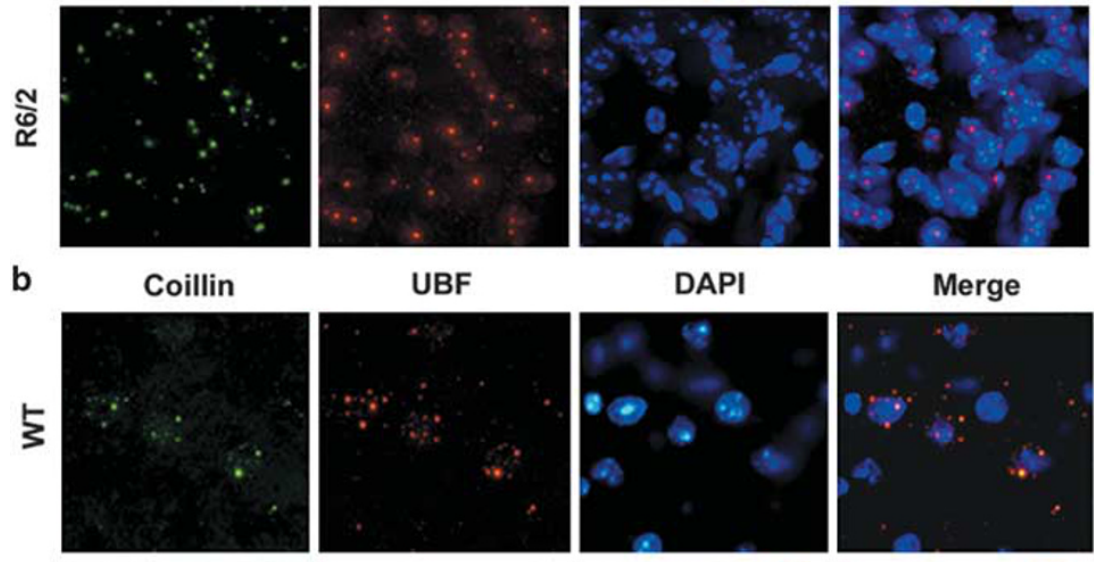

Merge
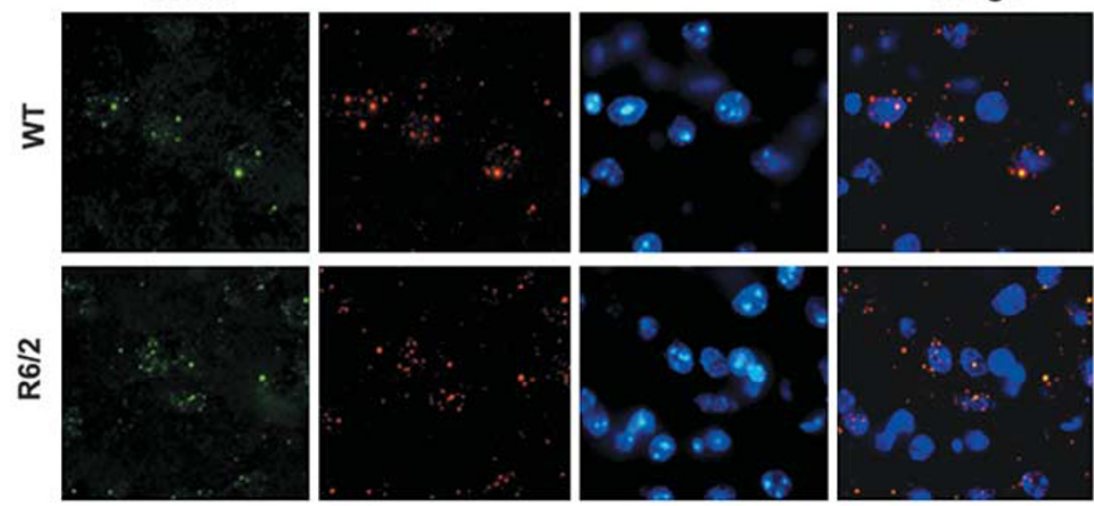

C
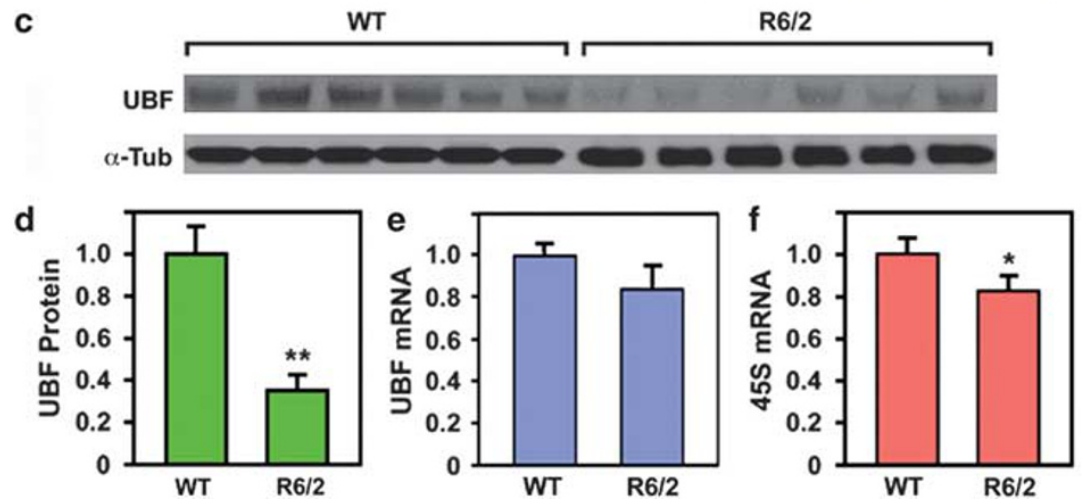

Figure 1 UBF1 is decreased in an animal model of HD (R6/2 line). (a) The immunoreactivity of UBF1 is markedly reduced in striatal neurons of R6/2 mice in comparison with wild type littermate control mice at 9 weeks of age. UBF1 and $\mathrm{mtH}$ tt are not co-localized. Scale bar: $10 \mu \mathrm{m}$. (b) Confocal microscopic analysis for UBF1 and coillin co-localization in the striatal tissue. Co-localization of coilin with UBF is decreased in striatal neurons of R6/2 mice in comparison with control mice. (c) Immunoblot assay for UBF-1 in the striatal tissue of WT and R6/2 mice. (d) Densitometry analysis of UBF1 protein level normalized to the levels of $\alpha$-tubulin ( $\alpha$-Tub). (e) qRT-PCR analysis of UBF1 mRNA shows no significant difference between WT and R6/2 mice. Densitometry analysis from WT $(n=6)$ and R6/2 $(n=6)$. UBF1 mRNA is normalized to GAPDH mRNA. (f) qRT-PCR analysis of 45S mRNA shows a difference between WT and R6/2 mice. *Significantly from wild type mice at $P<0.05 ;{ }^{* \star} P<0.01$. Scale bar: $10 \mu \mathrm{m}$

human HD gene with an expanded CAG repeat. ${ }^{9} \mathrm{R} 6 / 2$ mice develop brain and body weight loss at 6 weeks of age and a gait and movement disorder by 9-11 weeks. These clinical features, along with striatal atrophy and neuronal intranuclear inclusions, are similar to changes found in humans with HD. At 10 weeks of age, UBF1 levels in the striatum of $R 6 / 2$ mice were found to be significantly decreased compared with littermate controls as measured by immunofluorescence staining and confocal microscopy (Figures 1a and b) and Western blot analysis (Figure 1c). In addition, UBF1 levels in the striatum of human HD victims were markedly reduced compared with controls (Supplementary Figure 1A). The level of 18S RNA correlatively reduced in HD (Supplementary Figures $1 B$ and $C$ ). In contrast to protein level (Figure 1d), UBF1 mRNA levels in striatum were not significantly decreased in $\mathrm{R} 6 / 2$ mice compared with WT controls (Figure 1e). We also found that $48 \mathrm{~S}$ levels were downregulated in $\mathrm{R} 6 / 2$ mice in comparison with controls using quantitative real-time PCR (qRT-PCR) (Figure 1f). These results indicate that the UBF1 protein levels are reduced in HD mice and are linked to reducedribosomal transcription in vivo. 

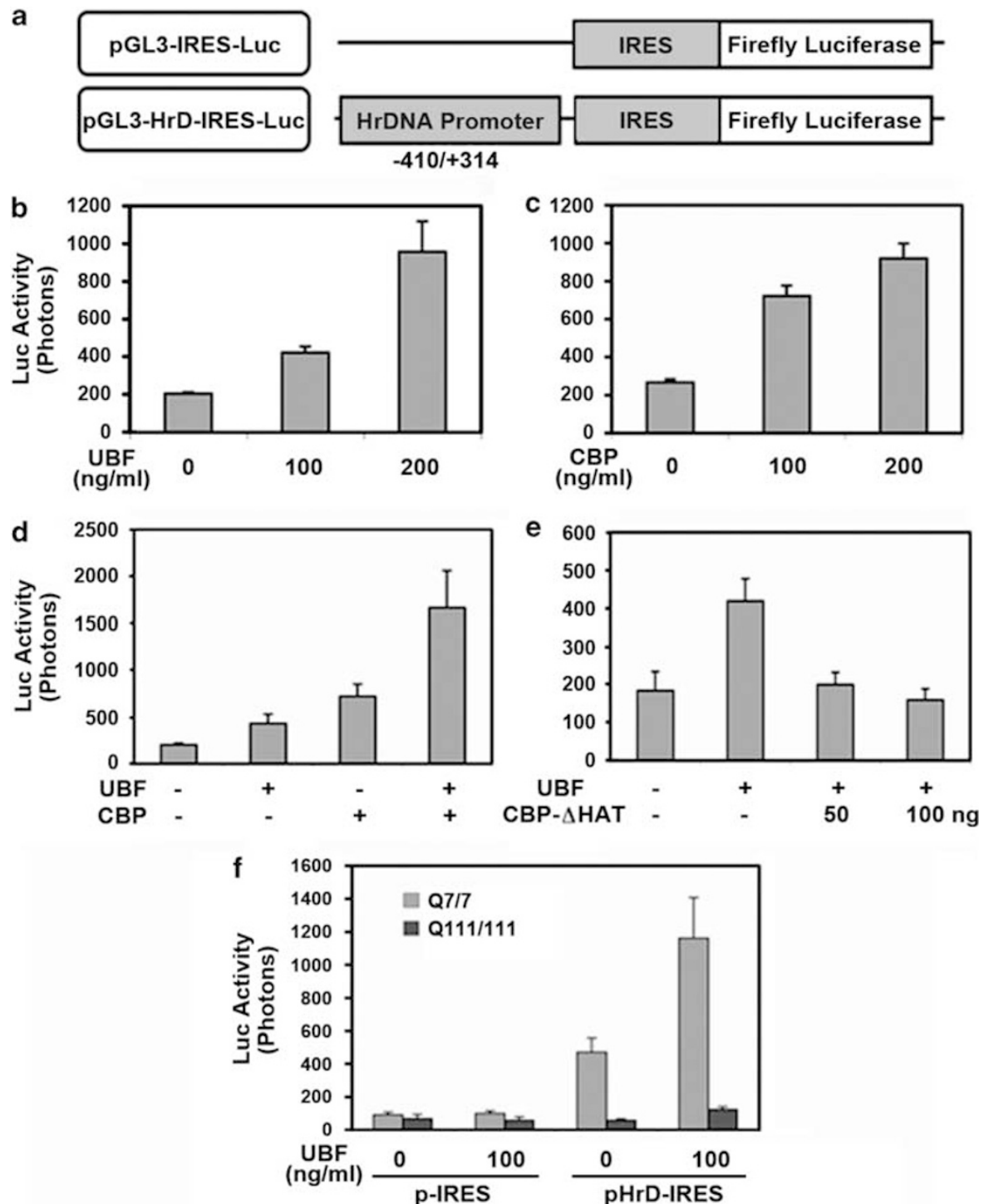

Figure 2 UBF1-dependent rDNA transcription is impaired in mutant HD cells. (a) The scheme of HrDNA IRES-reporter constructs. (b) UBF1 increases pHrD-IRES promoter activity in a dose-dependent manner. STHdh ${ }^{Q 7 / 7}(Q 7)$ cells were transiently cotransfected with $400 \mathrm{ng}$ of pHrD-IRES luciferase reporter plasmid and the other indicated constructs. Cells were incubated for $48 \mathrm{~h}$ and luciferase activity was measured. Transactivation of pHrD-IRES by UBF1 is increased in a dose-dependent manner. (c) CBP increases pHrD-IRES promoter activity in a dose-dependent manner. (d) UBF1 and CBP synergistically stimulate pHrD-IRES promoter activity. (e) The loss of acetyltransferase activity of CBP reduces UBF1-dependent transactivation of $\mathrm{pHrD}$-IRES promoter. UBF1 was cotransfected with a CBP mutant with HAT domain deletion (CBP-dHAT). (f) The basal rDNA transcriptional activity was downregulated in STHdh ${ }^{\mathrm{Q} 11 / 111}$ (Q111) cells. The data represent an average of three independent experiments

UBF1-induced rDNA transcription is impaired in a cellular model of HD. In order to examine how the transcriptional activity of rDNA is modulated by UBF1, we transiently transfected luciferase reporter vectors containing pIRES (internal ribosome entry segment) or pHrD (human rDNA)-IRES with UBF1, and measured the change of luciferase activity (Figure 2a). As expected, UBF1 increased in pHrD-IRES promoter activity in a dosedependent manner in STHdh ${ }^{\mathrm{Q} / 7}$ (Q7) cells (Figure 2b). As CBP can modulate UBF1 activity, we further elucidated how the interplay between UBF1 and CBP affects the regulation of rDNA transcription. We found that CBP enhanced rDNA transcription in a dose-dependent manner (Figure 2c). When UBF and CBP constructs were co-transfected, a synergistic effect on rDNA transcription was found (Figure 2d).
To confirm whether CBP-dependent and UBF1-induced rDNA transcription was due to the HAT activity of CBP, we used a CBP mutant construct with a mutant HAT site (CBPdHAT). As we predicted, co-transfection of UBF1 and mutant CBP-dHAT lead to the loss of the synergistic effect on the rDNA transcription (Figure 2e). The loss of CBP-HAT activity reduced the promoter activity of $\mathrm{pHrD}-\mathrm{IRES}$ to basal levels. UBF knockdown by shRNA reduced CBP-induced rDNA transcription (Supplementary Figure 2). We then examined transcriptional activity of rDNA in a cell line model of HD using wild type $\left(\mathrm{STH} d h^{\mathrm{Q} 7 / 7}\right)$ and mutant $\left(\mathrm{STH} d h^{\mathrm{Q} 111 / 111}\right.$ ) Huntingtin-expressing cells of striatal origin (Figure 2f). Basal rDNA transcriptional activity was higher in cells expressing $\mathrm{STHdh} h^{\mathrm{Q} 7 / 7}$ (Q7) and downregulated in $\mathrm{STH} d h^{\mathrm{Q} 111 / 111}$ (Q111) cells. UBF1-dependent rDNA transcription was 
dramatically impaired in mutant Q111 cells in contrast to Q7 cells, where UBF1 led to increased pHrD-IRES promoter activity in a dose-dependent manner. Using a dual luciferase assay (HrD-IRES fire fly luciferase versus Renilla luciferase), we found that the transcriptional system is dysregulated in Q111 cells compared with Q7 cells (Supplementary Figure 3). UBF1 knockdown using shRNA only reduced basal pHrD-IRES promoter activity in WT cells.
CBP dysregulation is linked to UBF1 dysfunction in HD. As CBP is known to modulate UBF1 and CBP and is dysregulated in $\mathrm{HD},{ }^{10}$ we sought to determine the relationship between CBP and UBF1 activity in HD. First, we confirmed that CBP levels are affected in HD mice and $\mathrm{HD}$ cell lines. CBP levels in the striatum and hippocampus of $\mathrm{R} 6 / 2$ mice were significantly reduced compared with littermate controls at 10 weeks of age, as monitored by western blot analysis (Figure 3a). Punctate CBP
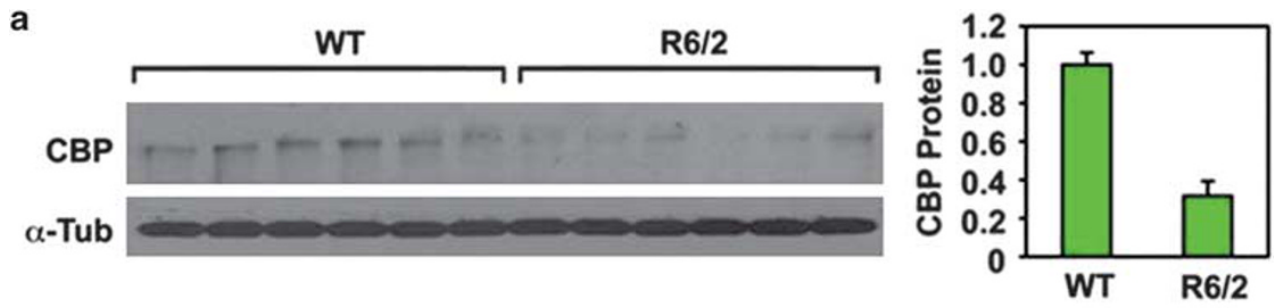

b
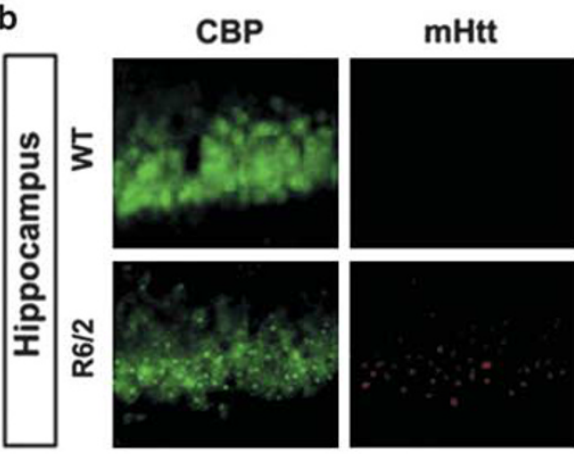

d
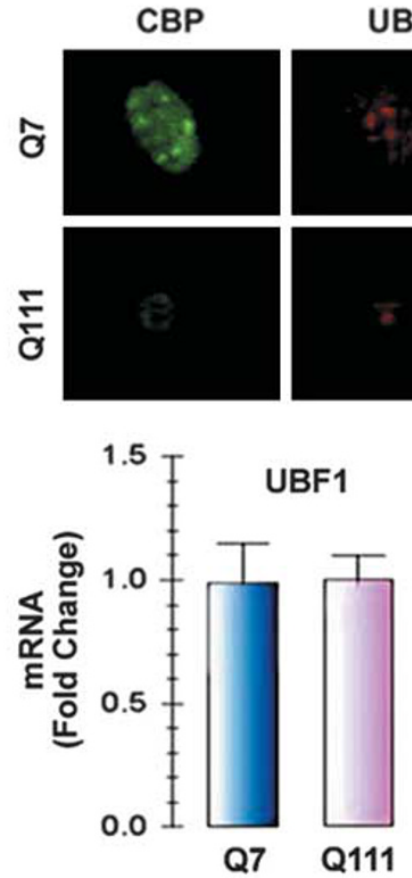
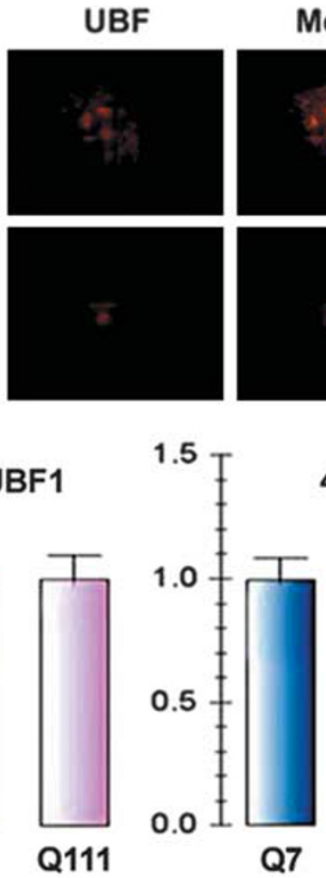

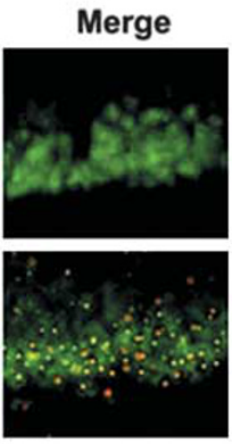

c
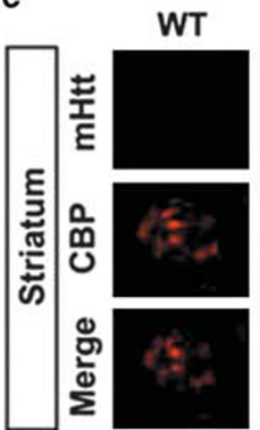

$\mathrm{R} 6 / 2$

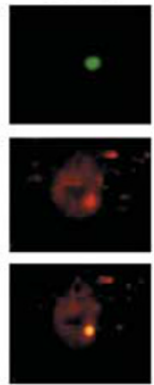

Q7 Q111

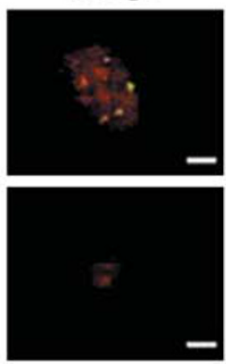

45S

e
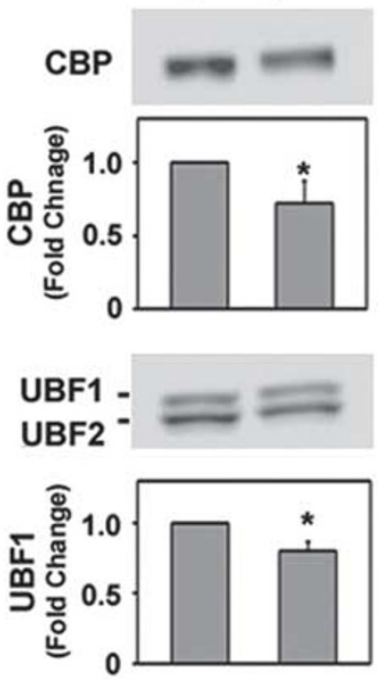

$\beta$-actin

Figure $3 \mathrm{CBP}$ is impaired in HD mice and cell line (Q111). (a) The protein level of CBP is markedly reduced in striatal neurons of R6/2 mice compared with wild type littermate control mice at 9 weeks of age. (b) CBP and mtHtt are co-localized in hippocampal neurons of R6/2 mice, but not in WT mice. (c) Confocal microscopic analysis for $\mathrm{mtH} H \mathrm{tt}$ and CBP co-localization in striatal neurons of R6/2 mice. Co-localization of $\mathrm{mtH} H \mathrm{tt}$ with $\mathrm{CBP}$ is increased in striatal neurons of R6/2 mice in comparison to control mice. (d) CBP and $\mathrm{mtH} H \mathrm{tt}$ are co-localized in Q7 cells and its colocalization is decreased in Q111 cells. (e) Immunoblot assay for CBP, UBF1 and $\beta$-actin in Q7 and Q111 cells. The data represent an average of 10 independent experiments. ${ }^{*}$ Significantly from the control at $P<0.05$. (f) qRT-PCR analysis of UBF1 mRNA and 45S in Q7 and Q111 cells. UBF1 mRNA and 45S were normalized to GAPDH mRNA. The data represent an average of three independent experiments 
a

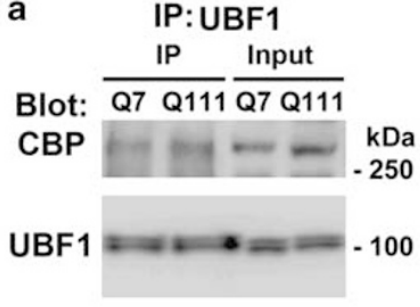

c
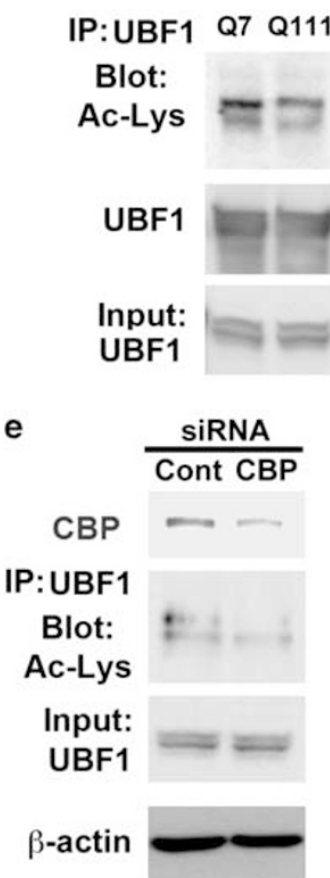

UBF1

Input: UBF1

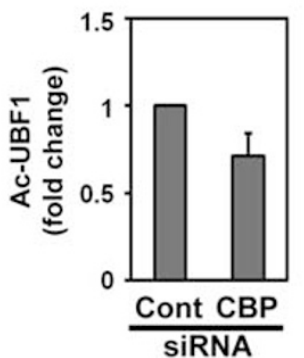

b
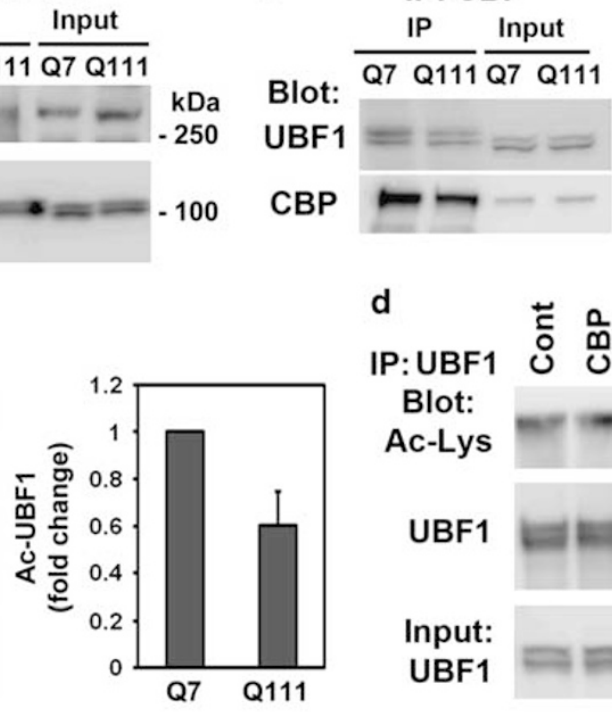

IP: UBF1 苍怘 兵

Blot:

Ac-Lys

UBF1

Input:

UBF1

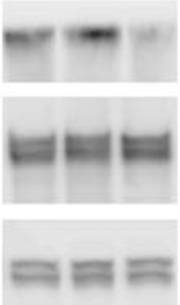

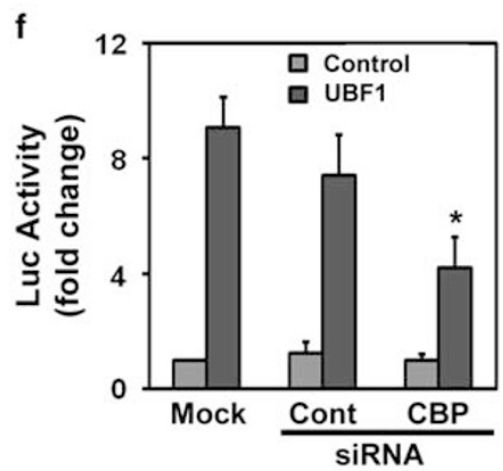

Figure 4 UBF1 interacts with CBP and its acetylation is modulated by CBP-HAT activity. (a) UBF1 interacts with CBP in intact cells. Cell lysates were immunoprecipitated with UBF1 and subsequently blots were probed with anti-CBP antibody. The same blots were then stripped and reprobed with anti-UBF1 antibody. (b) CBP is also immunoprecipiated with UBF1. (c) The acetylated level of UBF1 is reduced in Q111 cells in comparison with Q7 cells. (d) HAT deletion in CBP abrogates the acetylation of UBF1. Cells were transiently transfected with pCMV-WT-CBP or pCMV-CBP-dHAT mutant. The UBF1 was immunoprecipitaed and the blot was probed with anti-Ac-Lys antibody. (e) Knockdown of CBP by siRNA reduces the acetylation of UBF1. (f) Knockdown of CBP reduces UBF1-induced transcriptional activity of rDNA. ${ }^{*}$ Significantly from the control at $P<0.05$

immunoreactivity colocalized with $\mathrm{mtH} t \mathrm{tt}$ in neuronal nuclear inclusions in R6/2 mice (Figures $3 b$ and c). CBP and UBF1 protein levels were reduced in mutant Q111 cells compared with WT Q7 cells (Figures 3d and e) paralleling our in vivo findings. We examined the basal stability of UBF1 and CBP in intact cells, and determined whether CBP modulates UBF1 stability or not (Supplementary Figure 4). There was no difference in the stability of UBF1 and CBP in Q7 and Q111 cells, respectively. We did find, however, that ectopic expression of CBP increases the stability of UBF1. qRTPCR analysis showed decreased 45S mRNA in mutant Q111 cells (Figure 3f), indicating impairment of ribosomal biosynthesis similar to that we found in R6/2 mice.

Next, we further characterized the in vivo association of UBF1 and CBP by performing immunoprecipitations (IPs) on neuronal lysates, using either anti-UBF1 or anti-CBP antibodies. A prominent $300 \mathrm{kDa}$ band of $\mathrm{CBP}$ protein was present in UBF1 IPs (Figure 4a). The association of UBF1 and CBP was apparent in both Q7 and Q111 cells (Figure 4a).
We also confirmed the association between CBP and UBF1 using reverse IP with CBP antibody (Figure $4 b$ ). These results indicate that both UBF and CBP constitutively interact to generate rDNA transcription complex formation in neurons. Immunoblotting with anti-UBF1 antibody of anti-UBF1 IP verified that the same amounts of UBF1 were recovered by IP under different conditions. To test whether the acetylation status of UBF1 was altered in HD cells, we detected acetylated UBF1 (Ac-UBF1) using an anti-Ac-Lys antibody on UBF1 IPs. Interestingly, we found that Ac-UBF1 levels were reduced ( $>40 \%$ ) in mutant Q111 cells compared with WT Q7 cells (Figure 4c). We then addressed whether acetylation of UBF1 by CBP-HAT activity is responsible for CBP-dependent UBF1 transcriptional activation. Indeed, UBF1 acetylation was significantly augmented by WT CBP in $Q 7$ cells, but not by the CBP-dHAT mutant, as determined by IP using a UBF1 antibody, followed by immunoblotting using acetyl lysine antibody (Ac-UBF1) or UBF1 antibody alone (Figure $4 d$ ). CBP knockdown using siRNA CBP reduced 
a
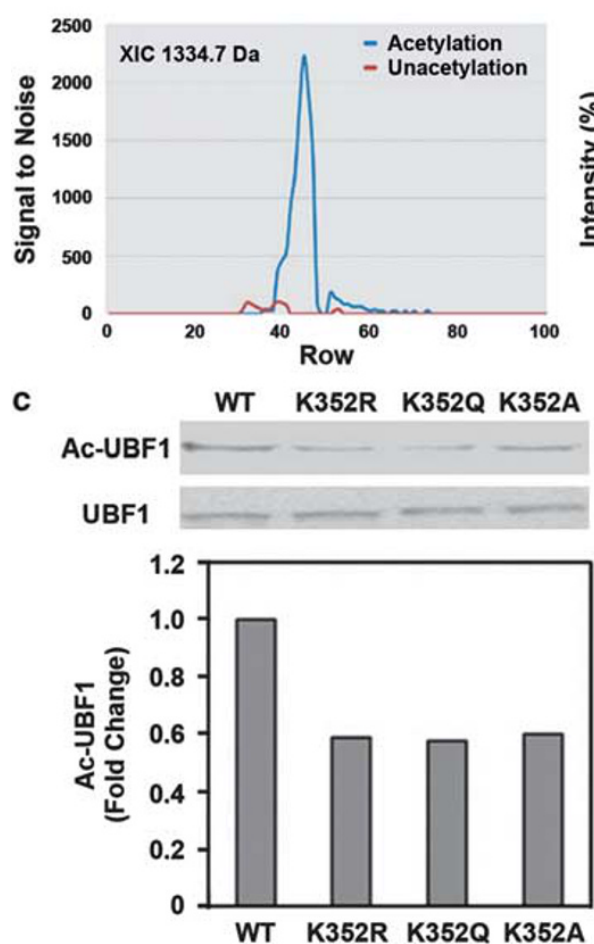

b

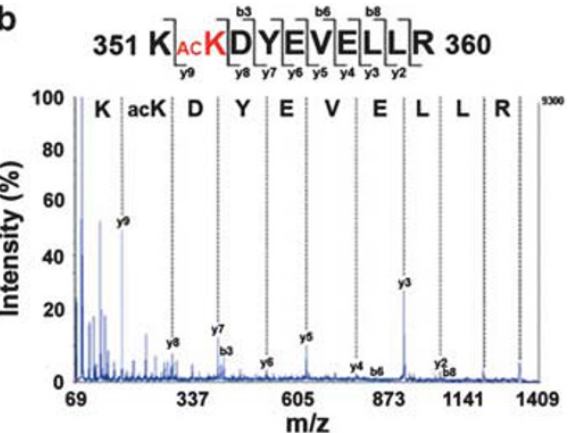

d

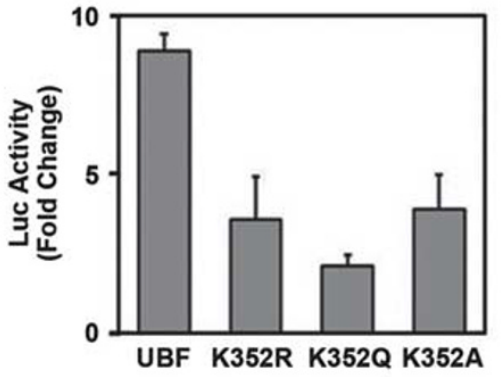

Figure 5 UBF1 is acetylated at K352 residue by CBP, and UBF1 acetylation site mutants (K352A, K352Q, and K352R) decreases the transcriptional activity of rDNA. (a) Comparison of LC-MALDI-MS/MS extracted ion chromatogram between in vitro-acetylated UBF1-HMG3 domain peptide (acetylation) and untreated control UBF1-HMG3 domain peptide (unacetylation). The extracted ion chromatogram (XIC) of the precursor ion at $\mathrm{m} / \mathrm{z} 1334.7 \mathrm{Da}$ was plotted as signal to nose (vertical) versus spot number (horizontal). (b) MALDI-TOF/TOF MS/MS spectrum of acetylated peptide KacKDYEVELLR (1334.7 m/z) from human UBF1 HMG3 domain (accession number: P17480). The chart represents $\mathrm{m} / \mathrm{z}$ (horizontal) versus intensity (vertical). (c) Mutation of UBF1 at K352 to A, Q, and R reduced its acetylation level in neuronal cells. pCMV-Flag-UBF1 s (WT, K352A, K352Q, and K352R) were transiently transfected, immunoprecipitaed with anti-Flag antibody and blotted with anti-Ac-Lys antibody. (d) UBF1 acetylation site mutants (K352A, K352Q, and $\mathrm{K} 352 \mathrm{R}$ ) decreases the transcriptional activity of rDNA in response to CBP

UBF1 acetylation levels, but the basal acetylation levels of UBF1 were not changed by siRNA control treatment (Figure 4e). In addition, CBP knockdown by siRNA markedly decreased UBF1-induced transcriptional activity of rDNA, compared with the siRNA control (Figure 4f).

UBF1 is acetylated at K352 by CBP. To identify which lysine (Lys) residue of UBF1 is directly acetylated by CBP, we performed an in vitro acetylation assay using GSTUBF1-HMG1-6 proteins and GST-CBP protein. As we found that GST-UBF1-HMG3 domain is specifically acetylated by CBP (Supplementary Figure 5), acetylated GST-UBF1HMG3 protein was pooled and cut by prescission enzyme for LC-MS/MS analysis. As shown in Figure 5, we identified that the Lys $(\mathrm{K}) 352$ of UBF1 was specifically acetylated by CBP (Figures $5 a$ and $b$ ). To further confirm whether the acetylation of UBF1 at K352 is critical for the transcriptional activation of rDNA, we generated $\mathrm{K} 352$ acetylation site mutants of UBF1 using site-directed mutagenesis. We co-transfected UBF1 acetylation site mutants (K352A, $\mathrm{K} 352 \mathrm{Q}, \mathrm{K} 352 \mathrm{R}$ ) with or without $\mathrm{CBP}$, and checked the acetylation status of UBF1 using IP and Ac-lys blot analysis. Three acetylation site mutants of UBF1 (K352A, K352Q, K352R) showed a marked reduction of acetylation by CBP
(Figure $5 \mathrm{c}$ ). These results suggest that UBF1 can be directly acetylated at $\mathrm{K} 352$ by $\mathrm{CBP}$ in intact neuronal cells. Concurrent with the reduced level of UBF1 acetylation in western blot analysis (Figure $5 \mathrm{c}$ ), acetylation site mutants (K352A, K352Q, K352R) decreased the transcriptional activity of rDNA as determined by reporter assay (Figure $5 d$ ).

The occupancy of UBF1 to the rDNA and ribosomal biosynthesis is altered in HD. Chromatin immunoprecipitation (ChIP) assays were performed to detect whether the UBF1 occupancy of DNA was altered within the rDNA gene promoter. ${ }^{11}$ Q7 and Q111 cells were cross linked with formaldehyde, sonicated, and immunoprecipitated with anti-UBF1 and normal rabbit IgG (as a control for normalization) antibodies. Eluted DNAs were amplified with specific primer sets for mouse rDNA promoter region such as ENH, UCE, CORE, and ETS3 (Figure 6a and Supplementary Table 1). As we expected, the occupancy of UBF1 to ENH, UCE, CORE, and ETS3 regions of the rDNA promoter was decreased ( $>40 \%$ ) in Q111 cells. In contrast, little or no PCR product was generated in Q7 cells, or with normal rabbit IgG as negative control (Figure $6 \mathrm{~b}$ ). In order to examine whether ribosomal biosynthesis is altered in $\mathrm{HD}, \mathrm{Q7}$ and Q111 cells were pulsing labeled for $30 \mathrm{~min}$ with 
a
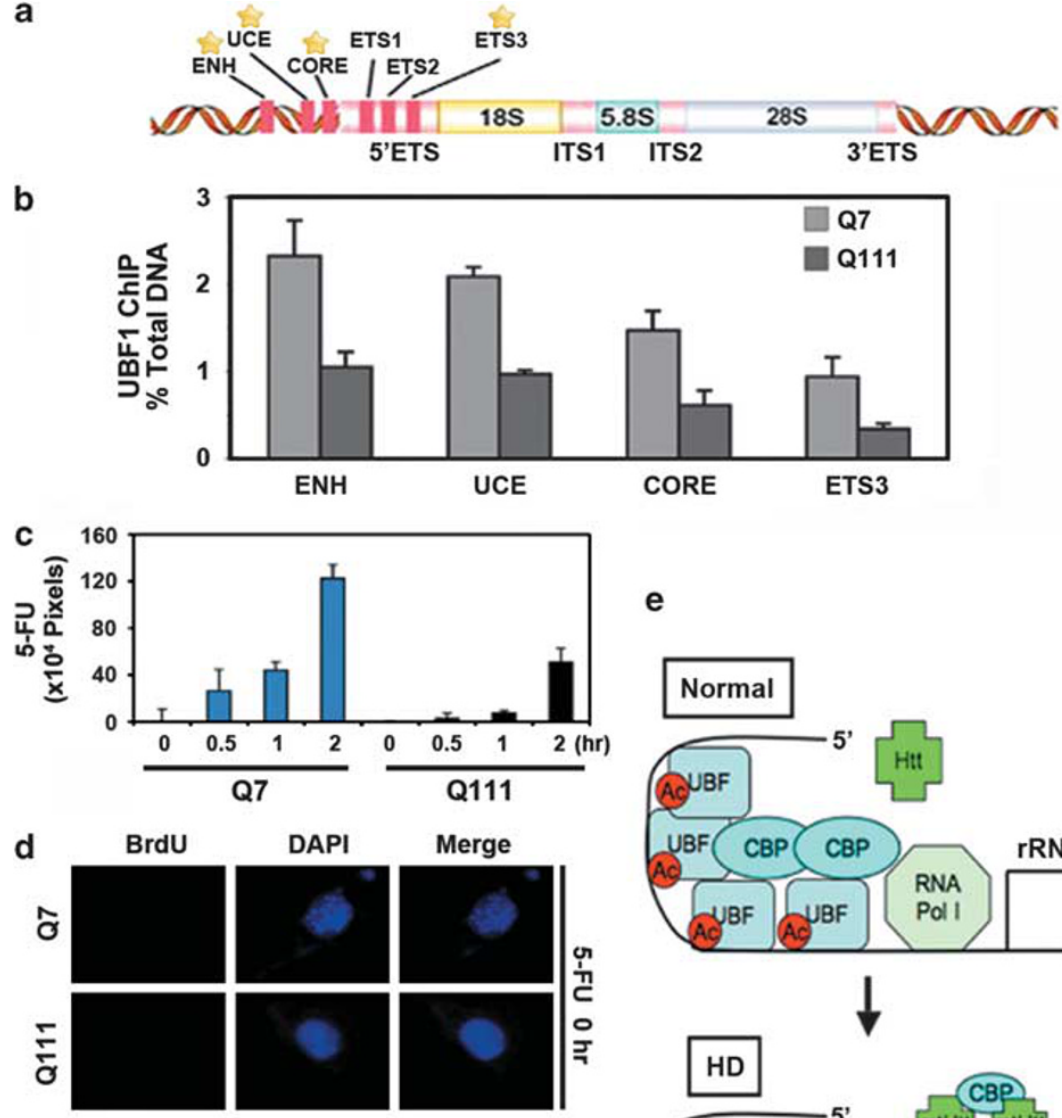

e
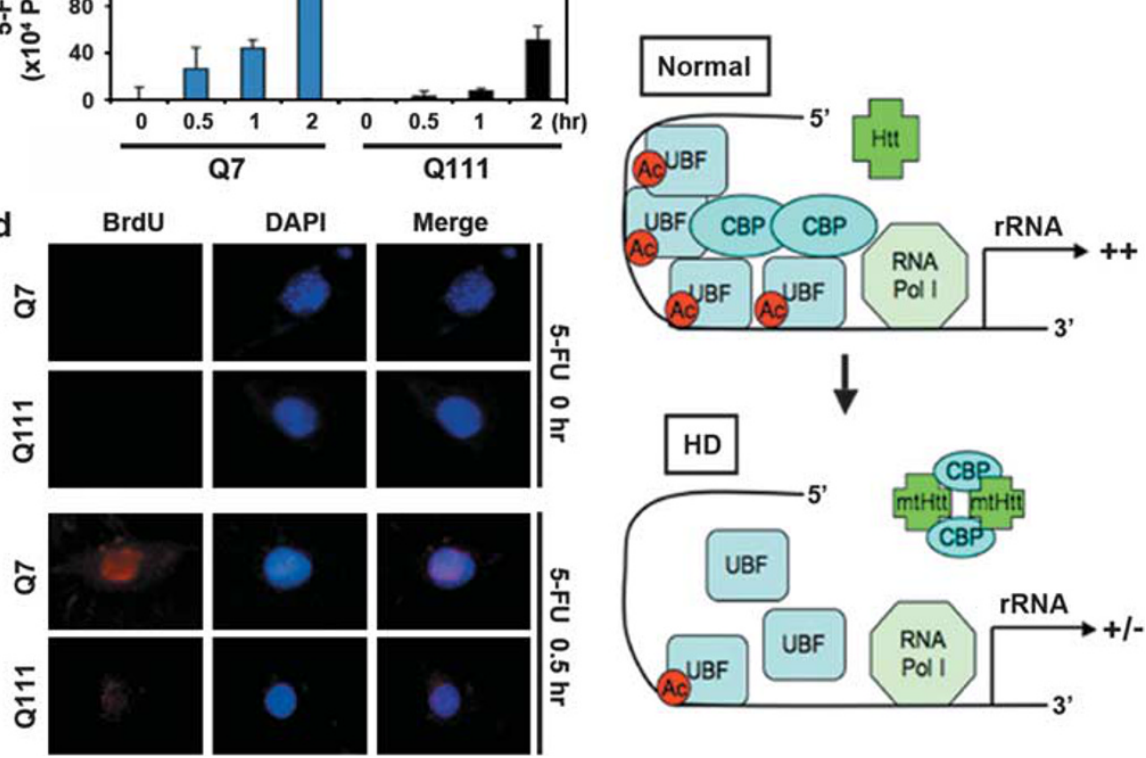

Figure 6 The occupancy of UBF1 to rDNA is changed in HD cell lines. (a) Positions of qRT-PCR primer set for detecting UBF1 occupancy in the promoter of mouse rDNA. (b) Quantitative ChIP analysis of UBF1 occupancy in the mouse rRNA gene. The UBF1 binding to rDNA was decreased in Q111 HD cells. Quantitative ChIP analysis of UBF1 binding to the rDNA (ENH, UCE, CORE, and ETS3) was performed as described in the 'Materials and Methods' section. (c) In situ transcription assay of 5'-FU incorporation into nucleolar rRNA in Q7 and Q111 cells. The level of $5^{\prime}$-FU incorporation was lowered in Q111 cells in comparison with Q7 cells. The time-dependent $(0.5,1$, and $2 \mathrm{~h}$ ) incorporation of halogenated nucleotide was detected by anti-BrdU antibody. The density (pixels) was averaged by counting five areas of each 60 cells. (d) Immunofluorescence staining images of Q7 and Q111 after 5'-FU incorporation. The nucleus was counterstained with DAPI. (e) A scheme of UBF1-dependent rDNA transcription in normal and HD condition. Normal condition (top): $\mathrm{CBP}$ interacts and acetylates UBF1 that is important in basal and constitutive transcription of rDNA (top). HD condition (bottom): mtHtt disrupts UBF1 and CBP complexes by sequestering CBP and decreases the acetylation of UBF1 at the proximal promoter of rDNA, subsequently leading to the repression of rDNA transcription

5-fluorouracil (FU) and immunolabeled with a mouse antiBrdU antibody to visualize nascent transcripts of nucleolus. Incorporation of 5 -FU was significantly decreased in mutant HD Q111 cells compared with WT Q7 cells (Figures 6c and d). These results confirm that the occupancy of UBF1 within rDNA gene loci is significantly reduced, which subsequently contributes to the dysregulation of rDNA transcription in HD. Figure 6e summarizes how CBP interacts and acetylates UBF1, which is important in basal constitutive and inducible expression of rDNA. In the context of $\mathrm{HD}, \mathrm{mtH}$ tt interferes with the ability of CBP to inhibit HAT activity and co-factor function. This in turn disrupts the interaction between UBF1 and CBP, and decreases the acetylation of UBF1 at the proximal promoter of rDNA to repress rDNA transcription.

\section{Discussion}

UBF is intimately involved in rDNA transcription as an RNA polymerase I specific transcription factor and is required for maintenance of rRNA genes to be transcriptionally active. ${ }^{12}$ UBF appears to be transcriptionally more active in specific neuronal cell type, such as sensory ganglia neurons. UBF consists of two polypeptides (UBF1 and 2), which form heteroand homodimers and arise from alternative splicing of a single transcript. ${ }^{13}$ UBF2 is five-fold less active than UBF1, ${ }^{14}$ and 
reasons why UBF2 has such poor transcriptional activity are unclear. UBF1 targeting to regions of heterochromatin is sufficient to induce large-scale chromatin decondensation. ${ }^{15}$ UBF1 binding throughout the rDNA gene repeat might therefore contribute to the formation of the active chromatin state of rDNA genes. ${ }^{16}$ In the current study, we found that UBF1 protein levels in the striatum were significantly decreased in R6/2 transgenic HD mice. We also found that the $45 \mathrm{~S}$ was downregulated in $\mathrm{R} 6 / 2$ mice in comparison with WT controls. These data indicate that abnormal protein level of UBF1 protein levels correlate with impaired ribosomal transcription in HD mice.

Post-translational modifications of UBF, such as acetylation and phosphorylation, have an important role in the control of rDNA transcription. ${ }^{17}$ Transcription factor acetylation is responsible for both activation and subnuclear localization. ${ }^{18}$ It has been well established that CBP has HAT activity that contributes to transcriptional activation by nuclear receptors. UBF acetylation is also important for cell cycle-dependent regulation of rRNA expression. It has been proposed that the transcription of rDNA is regulated upon the influence of two opposite processes through UBF acetylation by CBP and deacetylation by HDAC. ${ }^{10}$ In this context, CBP-dependent acetylation of UBF is linked to the transcription activation of rDNA. ${ }^{17}$ It is noteworthy that reduced HAT activity of CBP is thought to have a role in the toxic, transcriptional, and repressive effects of mutated proteins with expanded polyglutamine repeats such as $\mathrm{Htt}$ and atrophin. ${ }^{19-22}$ These findings, along with the established ability of UBF1-mediated rDNA transcription to be activated by acetyl transferases, ${ }^{23}$ raises the possibility that the neurodegenerative process in HD might be associated with changes in adaptive rDNA transcription caused by altered CBP acetyl transferase that affects the balance of acetylated and deacetylated UBF1. Indeed, we found that the acetylation levels of UBF1 are significantly lowered due to dysregulation of CBP function in both HD cells and HD mice. Our data shows that UBF1 and CBP interact in intact neurons. Reduced levels of acetylated UBF1 correlate with the reduced CBP level and activity in HD. We also directly demonstrated that the HAT activity of CBP controls UBF1 acetylation, using a HAT domain deletion mutant of CBP that caused a marked reduction of UBF1 acetylation.

In order to identify the specific acetylation site of UBF1 by CBP acetyl transferase, we performed LC-MALDI-MS/MS analysis for the in vitro-acetylated UBF-HMG protein by CBP. We confirmed the ability of CBP to acetylate UBF1 at K352 that resides in the HMG3 domain. Mutations at K352 to alanine $(A)$, glutamine $(Q)$, and arginine $(R)$ abrogated the acetylation of UBF1, as well as the transcriptional activation of UBF1 in response to CBP. These data prove that the CBPdependent acetylation of UBF1 has a pivotal role in rDNA gene transcription, ${ }^{17,24}$ and that CBP-dependent UBF1induced rDNA expression is disrupted in HD models. It is unclear, however, whether $\mathrm{mtHtt}$ and HD-related cellular changes modulate the association of HDAC to the UBF1 complex. It will be important to define precisely how UBF1 protein is deacetylated in HD in order to more fully understand the role of neuronal rDNA transcription and ribosomal synthesis in HD pathogenesis.
Deacetylase inhibitors, such as trichostatin $A$, that enhance UBF acetylation have been show to enhance rDNA gene expression. ${ }^{7,10}$ In the present study, we found that sodium butyrate triggered dose-dependent stimulation of rDNA transcription in mutant HD Q111 cells and WT Q7 cells in the presence of UBF1 (Supplementary Figure 6). We previously showed that enhanced transcription factors acetylation using three structurally distinct HDAC inhibitors improves neuronal survival in response to oxidative stress. ${ }^{25}$ The results of these studies are consistent with a model in which altered rDNA transcription is an additional mechanism contributing to the protective effects of HDAC inhibitors in neurons. Several groups have now reported the neuroprotective effects of HDAC inhibitors in combating the toxicity of mutant huntingtin or mutant androgen receptors, proteins with expanded polyglutamine repeats in vitro ${ }^{26,27}$ and in vivo. ${ }^{3}$ Future studies will be needed, however, to clarify the contribution of UBF1 acetylation and rDNA transcription to the neuroprotective effects of HDAC inhibitors.

In summary, our in vitro and in vivo findings show that $\mathrm{CBP}$ acetylates UBF1 at K352 to enhance the transcriptional activity of rDNA. Our results also indicate that dysregulation of UBF1 and CBP is linked to impaired rDNA transcription in HD. An HDAC inhibitor enhanced rDNA transcription in mutant HD cells. These data suggest that modulation of CBP-dependent UBF1-mediated rDNA synthesis may be a therapeutic target to improve neuronal rDNA transcription in HD by blunting the deleterious effects of expanded polyglutamine repeats.

\section{Materials and Methods}

Plasmid constructs. pIRES-Luc and human rRNA-luciferase vector (pHrD-IRES-Luc) was generously provided by Dr. Samson T. Jacob (Ohio State University). ${ }^{28}$ UBF1 acetylation mutants (K352A, K352Q, and K352R) were generated from $\mathrm{pCMV}$-Flag-UBF1 using site-directed mutagenesis kit (TOYOBO, Osaka, Japan) (Supplementary Table 1). pCMV-WT-CBP and pCMV-CBP-dHAT were previously described. ${ }^{11}$

In vitro protein acetylation assay. GST, GST-CBP, and GST-UBF1 HMG1-6 proteins were synthesized in Escherichia coli and purified with glutathione beads. Fusion proteins were eluted by using $20 \mathrm{~mm}$ glutathione in $1 \times$ HAT buffer (50 mM Tris- $\mathrm{HCl}$, pH 8.0, 10\% glycerol, $1 \mathrm{~mm}$ PMSF, $0.1 \mathrm{~mm}$ EDTA, $1 \mathrm{~mm}$ DTT, $0.05 \mathrm{M} \mathrm{NaCl}, 0.01 \mathrm{M}$ sodium butyrate (Calbiochem, Darmstadt, Germany)). The acetylation assay was performed as previously described. ${ }^{25}$

Cell culture and transfection assays. STHdh ${ }^{Q 7 / 7}$ (wild type) and STHdh ${ }^{Q 111 / 111}$ (HD knock-in striatal cell line expresses mutant huntingtin at endogenous level), were generously provided from Dr. Marcy MacDonald (Harvard Medical School). ${ }^{29}$ For transfection assays, $2.5 \times 10^{5}$ cells were plated onto 48 -well cell culture plates $24 \mathrm{~h}$ before transfection. DMRIE-C reagent (Invitrogen, Paisley, UK) has been used as a transfection reagent, and transfections were performed according to the manufacturer's protocol. When $\mathrm{NaB}$ was used in the experiments, the calculated amount of the HDAC inhibitor was added directly to the medium $24 \mathrm{~h}$ after transfection. The luciferase activity was measured and normalized to protein concentration. The Dual Luciferase Assay kit (Promega, Madison, WI, USA) was used to determine the difference of internal transcriptional activity between $Q 7$ and Q111 cells. Data represent the mean of three or more independent experiments.

Animals. Male $\mathrm{R} 6 / 2$ mice were bred with females from their background strain (B6CBAFI/J), and offspring were genotyped using PCR. ${ }^{9,30,31} \mathrm{CAG}$ repeat length remained stable within a 147-153 range. Female mice were used in the experimental paradigms.

Quantitative real time-PCR (qRT-PCR). Total RNA was isolated from cells using a commercial extraction system (Qiagen, Crawley, UK). A total of $1 \mu \mathrm{g}$ 
RNA has been used for CDNA preparation with iScript cDNA Synthesis Kit (BioRad) according to manufacturer's protocols. CDNA from each sample was amplified by RT-PCR using iQ SYBR Green Supermix (BioRad, Hercules, CA, USA). RNA quantities were normalized using GAPDH mRNA as a reference. ${ }^{11}$ PCR cycling conditions were: denaturation for $3 \mathrm{~min}$ at $95^{\circ} \mathrm{C}$, then 40 cycles of amplification for $15 \mathrm{~s}$ at $95^{\circ} \mathrm{C}, 15 \mathrm{~s}$ at $60^{\circ} \mathrm{C}, 20 \mathrm{~s}$ at $70^{\circ} \mathrm{C}$, followed with $30 \mathrm{~s}$ at $72^{\circ} \mathrm{C}$. For melt curve, data collection has been used 33 cycles, $6 \mathrm{~s}$ each, with the temperature increased from $60^{\circ} \mathrm{C}$ to $92^{\circ} \mathrm{C}$ (increase set point temperature after cycle 2 by $1^{\circ} \mathrm{C}$ ).

Chromatin immunoprecipitation (ChIP). ChIP for UBF1 binding to rDNA was performed using a CHIP assay kit (Upstate Biotechnology, Billerica, MA, USA) as described previously. ${ }^{11,25,32}$ Q7 and Q111 cells were crosslinked with $1 \%$ formaldehyde for $20 \mathrm{~min}$ at room temperature. The lysates were sonicated six times, with each time for $30 \mathrm{~s}$ using Bioruptor (Diogenode, Denville, NJ, USA). After centrifugation, the supernatant was diluted in ChIP dilution buffer, and then incubated overnight at $4^{\circ} \mathrm{C}$ with anti-UBF1 antibody. qPCR primers were shown on the Supplementary Table 2.

In-gel trypsin digestion and off-line 2 dimensional LC-MALDIMS/MS analysis. In-gel trypsin digestion and Nano LC separations were performed as previously reported, with some minor modifications. ${ }^{32,33,34}$ The details of method are on line Supplementary Materials and Methods.

Confocal microscopy. Indirect labeling methods were used to determine the UBF-1 (Santa Cruz Biotechnology, Santa Cruz, CA, USA) (1:200), coillin (Chemicon) (1:200), mtHtt (Chemicon, Temecula, CA, USA) (1:1000), and CBP (Santa Cruz Biotechnology) $(1: 200)$. The procedures were performed as previously described. ${ }^{31}$ Images were analyzed using a spinning disk confocal microscope (Olympus DSU, Tokyo, Japan). Deconvolution and 3-dimensional construction of the confocal image was performed by AQI-X-COMBO-CWF program (Media cybernetics Bethesda, MD, USA). Control experiments were performed in the absence of primary antibody or in the presence of blocking peptide. ${ }^{31}$

Western blot analysis. Western blot was performed as previously described. ${ }^{30,35,36}$ A total of $30 \mu \mathrm{g}$ of protein was subjected to SDS-PAGE $(10 \%)$, and blotted with anti-UBF1 (Upstate Biotechnology), anti-CBP (A-22: sc-369 and C-20: sc-583) (Santa Cruz Biotechnology), and anti-huntingtin (C-20: sc-351) (Santa Cruz Biotechnology) antibody. Protein loading was controlled by probing for $\alpha$-tubulin (Sigma, Gillingham, UK) or beta-actin (Santa Cruz Bitotechnology) on the same membrane.

RNA interference experiment. Q7 and Q111 $\left(1 \times 10^{5}\right.$ cells $\left./ \mathrm{ml}\right)$ or SHSY5Y $\left(2 \times 10^{5}\right.$ cells $\left./ \mathrm{ml}\right)$ were transiently transfected with 100 to $400 \mathrm{nM}$ of Stealth control RNAi and CBP RNAi (Invitrogen Life Technology, Carlsbad, CA, USA) and control shRNA and UBF1 shRNA using DMRIE-C transfection reagent (Invitrogen Life Technology) in the presence or absence of HrD-IRES reporter for $48 \mathrm{~h}^{11}$

Run-on transcription assay and immunofluorescence. Nascent RNA was detected using a method previously described, with slight modifications. ${ }^{4}$ Briefly, cells were incubated with $5 \mathrm{~mm} \mathrm{5-FU} \mathrm{(Sigma)} \mathrm{for} \mathrm{indicated} \mathrm{time,} \mathrm{and} \mathrm{fixed}$ with $4 \%$ paraformaldehyde for $30 \mathrm{~min}$ at $4{ }^{\circ} \mathrm{C}$. Incorporated 5 -FU was labeled with anti-BrdU antibody (Serotec, Oxford, UK) for overnight at $4^{\circ} \mathrm{C}$, and with DyLight594-conjugated anti-mouse IgG antibody (Jackson Laboratories, Bar Harbor, ME, USA) for $1 \mathrm{~h}$. The nuclei were counterstained with DAPI, and labeled signals were visualized using fluorescent microscopy (Olympus, Tokyo, Japan). For the quantitative analysis, emitted signals for 5-FU was captured and their intensities were analyzed using multigauge program (Fuji, Tokyo, Japan).

\section{Conflict of interest}

The authors declare no conflict of interest.

Acknowledgements. We thank Dr. Samson T. Jacob for pIRES-Luc and Human rRNA-luciferase Vector and Dr. Marcy MacDonald for STHdh ${ }^{Q 7 / 7}$ and STHdh ${ }^{Q 111 / 111}$ cells. We thank Dr. Byoung Yul Soh for his technical assistance. This study was supported by WCU Neurocytomics Program Grant (800-20080848) (H.R.) and SRC Grant (2010-0029-403) (H.R.) from KOSEF and NIH NS52724 (H.R.). This work was also supported in part by the Converging Research Center Program (20090094081) (Y.K.) through NRF of Korea.
1. Vonsattel JP, DiFiglia M. Huntington disease. J Neuropathol Exp Neurol 1998; 57: 369-384.

2. Sadri-Vakili G, Cha JH. Mechanisms of disease: Histone modifications in Huntington's disease. Nat Clin Pract Neurol 2006; 2: 330-338.

3. Steffan JS, Pallos J, Poelman M, McCampbell A, Apostol BL, Kazantsev A et al. Histone deacetylase inhibitors arrest polyglutamine-dependent neurodegeneration in Drosophila. Nature 2001; 413: 739-743.

4. Casafont I, Bengoechea R, Navascués J, Pena E, Berciano MT, Lafarga M. The giant fibrillar center: a nucleolar structure enriched in upstream binding factor (UBF) that appears in transcriptionally more active sensory ganglia neurons. J Struct Biol 2007; 159: 451-461.

5. Lafontaine DL, Tollervey D. The function and synthesis of ribosomes. Nat Rev Mol Cell Biol 2001; 2: 514-520.

6. Akhmanova A, Verkerk T, Langeveld A, Grosveld F, Galjart N. Characterisation of transcriptionally active and inactive chromatin domains in neurons. J Cell Sci 2000; 113: 4463-4474.

7. McStay B, Grummt I. The epigenetics of rRNA genes: from molecular to chromosome biology. Annu Rev Cell Dev Biol 2008; 24: 131-157.

8. Hirschler-Laszkiewicz I, Cavanaugh A, Hu Q, Catania J, Avantaggiati ML, Rothblum LI. The role of acetylation in rDNA transcription. Nucleic Acids Res 2001; 29: 4114-4124.

9. Yamada M, Sato T, Shimohata T, Hayashi S, Igarashi S, Tsuji S et al. Interaction between neuronal intranuclear inclusions and promyelocytic leukemia protein nuclear and coiled bodies in CAG repeat diseases. Am J Pathol 2001; 159: 1785-1795.

10. Mangiarini L, Sathasivam K, Seller M, Cozens B, Harper A, Hetherington C et al. Exon1 of the $H D$ gene with an expanded $C A G$ repeat is sufficient to cause a progressive neurological phenotype in transgenic mice. Cell 1996; 87: 493-506.

11. Pelletier G, Stefanovsky VY, Faubladier M, Hirschler-Laszkiewicz I, Savard J, Rothblum LI et al. Competitive recruitment of $\mathrm{CBP}$ and Rb-HDAC regulates UBF acetylation and ribosomal transcription. Mol Cell 2000; 6: 1059-1066.

12. Lee J, Hagerty S, Cormier KA, Kim J, Kung AL, Ferrante RJ et al. Monoallele deletion of CBP leads to pericentromeric heterochromatin condensation through ESET expression and histone H3 (K9) methylation. Hum Mol Genet 2008; 17: 1774-1782.

13. Stefanovsky VY, Moss T. The splice variants of UBF differentially regulate RNA polymerase I transcription elongation in response to ERK phosphorylation. Nucleic Acids Res 2008; 36: 5093-5101.

14. O'Mahony DJ, Rothblum LI. Identification of two forms of the RNA polymerase I transcription factor UBF. Proc Natl Acad Sci USA 1991; 88: 3180-3184.

15. Hannan RD, Stefanovsky V, Taylor L, Moss T, Rothblum LI. Overexpression of the transcription factor UBF1 is sufficient to increase ribosomal DNA transcription in neonatal cardiomyocyte: implications for cardiac hypertrophy. Proc Natl Acad Sci USA 1996; 93: 8750-8755.

16. Chen D, Belmont AS, Huang S. Upstream binding factor association induces large-scale chromatin decondensation. Proc Natl Acad Sci USA 2004; 101: 15106-15111.

17. Sanij E, Poortinga G, Sharkey K, Hung S, Holloway TP, Quin J et al. UBF levels determine the number of active ribosomal RNA genes in mammals. J Cell Biol 2008; 183: 1259-1274.

18. Meraner J, Lechner M, Loidl A, Goralik-Schramel M, Voit R, Grummt I et al. Acetylation of UBF changes during the cell cycle and regulates the interaction of UBF with RNA polymerase I. Nucleic Acids Res 2006; 34: 1798-1806.

19. Lin $\mathrm{CH}$, Platt MD, Ficarro SB, Hoofnagle MH, Shabanowitz J, Comai L et al. Mass spectrometric identification of phosphorylation sites of rRNA transcription factor upstream binding factor. Am J Physiol Cell Physiol 2007; 292: 1617-1624.

20. Steffan JS, Kazantsev A, Spasic-Boskovic O, Greenwald M, Zhu YZ, Gohler $\mathrm{H}$ et al. The Huntington's disease protein interacts with p53 and CREB-binding protein and represses transcription. Proc Natl Acad Sci USA 2000; 97: 6763-6768.

21. Zoghbi HY, Orr HT. Glutamine repeats and neurodegeneration. Annu Rev Neurosci 2000; 23: 217-247.

22. McCampbell A, Fischbeck KH. Polyglutamine and CBP: fatal attraction? Nat Med 2001; 7 : 528-530.

23. Nucifora FC, Sasaki M, Peters MF, Huang H, Cooper JK, Yamada M et al. Interference by huntingtin and atrophin-1 with CBP-mediated transcription leading to cellular toxicity. Science 2001; 291: 2423-2428.

24. Doetzhofer A, Rotheneder H, Lagger G, Koranda M, Kurtev V, Brosch G et al. Histone deacetylase 1 can repress transcription by binding to SP1. Mol Cell Biol 1999; 19: 5504-5511.

25. Stefanovsky VY, Moss T. Regulation of rRNA synthesis in human and mouse cells is not determined by changes in active gene count. Cell Cycle 2006; 5: 735-739.

26. Ryu $\mathrm{H}$, Lee J, Olofsson BA, Mwidau A, Dedeoglu A, Escudero M et al. Histone deacetylase inhibitors prevent oxidative neuronal death independent of expanded polyglutamine repeats via an Sp1-dependent pathway. Proc Natl Acad Sci USA 2003; 100: $4281-4286$.

27. McCampbell A, Taye AA, Whitty L, Penney E, Steffan JS, Fischbeck KH. Histone deacetylase inhibitors reduce polyglutamine toxicity. Proc Natl Acad Sci USA 2001; 98: 15179-15184.

28. Hughes RE, Olson JM. Therapeutic opportunities in polyglutamine disease. Nat Med 2001; 7: 419-423.

29. Ghoshal K, Majumder S, Datta J, Motiwala T, Bai S, Sharma SM et al. Role of human ribosomal RNA (rRNA) promoter methylation and of methyl-CpG-binding protein MBD2 in the suppression of rRNA gene expression. J Biol Chem 2004; 279: 6783-6793. 
30. Trettel F, Rigamonti D, Hilditch-Maguire P, Wheeler VC, Sharp AH, Persichetti F et al. Dominant phenotypes produced by the HD mutation in STHdh(Q111) striatal cells. Hum Mol Genet 2000; 9: 2799-2809.

31. Ryu H, Lee J, Hagerty SW, Soh BY, McAlpin SE, Cormier KA et al. ESET/SETDB1 gene expression and histone H3 (K9) trimethylation in Huntington's disease. Proc Natl Acad Sci USA 2006; 103: 19176-19181.

32. Ryu H, Lee J, Zaman K, Kubilis J, Ferrante RJ, Ross BD et al. Sp1 and Sp3 are oxidative stress-inducible, anti-death transcription factors in cortical neurons. J Neurosci 2003; 23 : 3597-3606.

33. Ryu H, Lee J, Impey S, Ratan RR, Ferrante RJ. Antioxidants modulate mitochondrial protein kinase $A$ and increase CREB binding to D-Loop DNA of the mitochondrial genome in neurons. Proc Natl Acad Sci USA 2005; 102 : 13915-13920.

34. Kim T, Kim SJ, Kim K, Kang UB, Lee C, Park KS et al. Profiling of vitreous proteomes from proliferative diabetic retinopathy and nondiabetic patients. Proteomics 2007; 7: $4203-4215$.

35. Garaguso I, Borlak J. Matrix layer sample preparation: an improved MALDI-MS peptide analysis method for proteomic studies. Proteomics 2008; 8: 2583-2595.

36. Ryu H, Smith K, Camelo SI, Carreras I, Lee J, Iglesias AH et al. Sodium phenylbutyrate prolongs survival and regulates expression of anti-apoptotic genes in transgenic amyotrophic lateral sclerosis mice. J Neurochem 2005; 93: 1087-1098.

Supplementary Information accompanies the paper on Cell Death and Differentiation website (http://www.nature.com/cdd) 